\title{
A NONCOMMUTATIVE HILBERT BASIS THEOREM AND SUBRINGS OF MATRICES
}

\author{
BY \\ S. A. AMitsur( $\left.{ }^{1}\right)$
}

\begin{abstract}
A finitely generated central extension $A\left[u_{1}, \ldots, u_{k}\right]$ of a commutative noetherian ring $A$, satisfies the ascending chain condition for ideals $P$ for which $A\left[u_{1}, \ldots, u_{k}\right] / P$ can be embedded in matrix rings $M_{n}(K)$ over arbitrary commutative rings $K$ and $n$ bounded. The method of proof leads to an example of a ring $R$ which satisfies the same identities of $M_{n}(K)$ but nevertheless cannot be embedded in any matrix ring over a commutative ring of arbitrary finite order.
\end{abstract}

1. Introduction. Let $A$ be a commutative integral domain (with a unit), we shall use the following notations and constructions: $A[x]=A\left[x_{1}, x_{2}, \ldots, x_{k}\right]$ be the free ring generated by a set $\left\{x_{i}\right\}$ of noncommutative indeterminates which commute with the elements of $A$.

Denote by $\mathscr{M}_{n}$ the ideal of all polynomials $p[x] \in A[x]$ such that $p\left[x_{1}, \ldots, x_{k}\right]=0$ for all substitutions $x_{i}=P_{i}$ of $n \times n$ matrices in an arbitrary commutative ring $K$ containing $A$.

Let $\left\{\xi_{\lambda \mu}^{i}\right\} i=1, \ldots, k, \lambda, \mu=1,2, \ldots, n$ be $n^{2} k$ commutative indeterminates over $A$. Let $A[\xi]$ denote the ring of all commutative polynomials in the $\xi_{\lambda \mu}^{i}$ over $A$.

Consider the $k$ generic matrices $X_{i}=\left(\xi_{\lambda \mu}^{i}\right)$ in the ring $M_{n}(A[\xi])$ of all $n \times n$ matrices with entries in $A[\xi]$.

The subring of $M_{n}(A[\xi])$ generated by $A$ and the generic matrices $X_{i}$ will be denoted by $A[X]=A\left[X_{1}, \ldots, X_{k}\right]$. The injection $A[X] \rightarrow M_{n}(A[\xi])$ will be denoted by $i$ but often will be omitted as $A[X]$ will be considered as a subring of $M_{n}(A[\xi])$.

If $Q$ is any subset of $A[X]$ we shall denote by $\{Q\}$ the ideal generated in $M_{n}(A[\xi])$ by the set $Q$.

Procesi has shown in [2, Theorem 2.5] that the ring $A[X]$ for $A$ noetherian, as well as any finitely generated extension of $A$ which satisfies an identity-satisfies the ascending chain condition on semiprime ideals. This is an extension of one form of the famous Hilbert's basis theorem for the noncommutative case. In the first part of the present paper we follow Procesi's proof to show that the free ring

Received by the editors September 8, 1969.

AMS Subject Classifications. Primary 1649, 1648, 1625.

Key Words and Phrases. Hilbert basis theorem, generic matrices, ascending chain condition, finitely generated rings with identities.

( $\left.{ }^{1}\right)$ This research was partly supported by NSF grant GP-9572.

Copyright (C) 1970, American Mathematical Society 
$A\left[x_{1}, x_{2}, \ldots, x_{k}\right]$ will satisfy the ascending chain condition for sequences of ideals $P_{0} \subseteq P_{1} \subseteq \cdots \subseteq P_{n} \subseteq \cdots$ such that the rings $A[x] / P_{i}$ can be embedded in matrix rings $M_{n_{i}}\left(K_{i}\right)$ for commutative rings $K_{i}$ and where the set of integers $n_{i}$ is bounded. This probably cannot be extended much more. In fact we prove that even the ring $A[X]$ of generic matrices does not satisfy the ascending chain condition on twosided ideals. The method of proof of this result yields an example of a homomorphic image of a subring of the matrix ring $M_{n}(K)$ over a commutative ring $K(n \geqq 3)$ which satisfies exactly the identities of $M_{n}(A)$ (as well as of $M_{n}(K)$ ) and yet cannot be embedded in any finite matrix ring over any commutative ring.

2. The ascending chain condition. If $P$ is an ideal in the ring of generic matrices $A[X]$, we are interested in the problem of embedding the quotient ring $A[X] / P$ in a matrix ring $M_{n}(K)$, for some commutative $K$. We shall restrict ourselves only to embeddings as $A$-algebras, namely $K$ will be assumed (sometimes proved) to be an $A$-algebra and without any loss of generalization $K$ can be assumed to have a unit and, therefore, we have a canonical homomorphism $\tau_{0}: A \rightarrow K$ given by $\tau_{0}(a)=a \cdot 1$ for every $a \in A$.

Our fundamental lemma:

Lemma 1. The ring $A[X] / P$ can be embedded (as an A-algebra) in some matrix $M_{n}(K)$ over a commutative $A$-algebra $K$-if and only if $P$ satisfies:

$$
\{P\} \cap A[X]=P .
$$

Assume that there exist a monomorphism $j: A[X] / P \rightarrow M_{n}(K)$ for some commutative ring $K$, and as it was pointed out before we have a homomorphism $\tau_{0}: A \rightarrow K$ given by $\tau_{0}(a)=a \cdot 1$. Consider the following commutative diagram

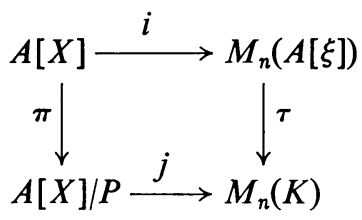

where $i$ is the inclusion $A[X] \subseteq M_{n}(A[\xi])$, and $\pi$ is canonical projection of $A[X]$ onto $A[X] / P$. The homomorphism $\tau$ is the extension of $\tau_{0}: A \rightarrow K$ determined as follows: if $j\left(X_{i}\right)=\left(t_{\lambda \mu}^{i}\right)=T_{i}$ is a matrix in $M_{n}(K)$ extend $\tau_{0}$ to a mapping $\tau_{1}: A[\xi] \rightarrow K$ by setting $\tau_{1}\left(\xi_{\lambda \mu}^{i}\right)=t_{\lambda \mu}^{i}$, and then extend $\tau_{1}$ to a homomorphism $\tau: M_{n}(A[\xi]) \rightarrow$ $M_{n}(K)$ by setting $\tau\left(p_{\lambda \mu}\right)=\left(\tau_{1}\left(p_{\lambda \mu}\right)\right)$. Clearly $\tau_{1}$ and $\tau$ are well defined and satisfy the commutativity condition $j \pi=\tau i$.

Now, clearly $\{P\} \cap A[X] \supseteq P$. To prove the inclusion in the other direction, we note that since $j \pi(P)=0$, it follows by the commutativity that $\tau i(P)=0$. This means that $i(P)=P$ is in the kernel of $\tau$, hence also the whole ideal $\{P\}$ in $M_{n}(A[\xi])$ generated by $P$ belongs to $\operatorname{Ker} \tau$. Thus, if $T \in\{P\} \cap A[X]$ then $\tau(T)=0$. Since $i(T)=T$ for $T \in A[X]$ we have $0=\tau i(T)=j \pi(T)$ and since $j$ is assumed to be a monomorphism, it follows that $\pi(T)=0$ i.e. $T \in \operatorname{Ker} \pi=P$. Q.E.D. 
Conversely, let $P$ satisfy the relation (*). The ideal $\{P\}=M_{n}(L)$ for some ideal $L$ in $A$ [ $\xi]$. This is the form of every ideal in $M_{n}(A[\xi])$ since $A$ contains a unit element. Thus, the injection $i: A[X] \rightarrow M_{n}(A[\xi])$ maps $P$ into $\{P\}$; hence $i$ induces a homomorphism $i_{p}: A[X] / P \rightarrow M_{n}(A[\xi]) /\{P\}$. It follows by $(*)$ that $i_{p}$ is actually a monomorphism since a class $q+P$ in $A[X] / P$ belongs to $\operatorname{Ker} i_{p}$ if and only if $q \in\{P\}$ which is equivalent to $q \in\{P\} \cap A[X]=P$ by (*). Finally

$$
M_{n}(A[\xi]) /\{P\}=M_{n}(A[\xi]) / M_{n}(L) \cong M_{n}(K)
$$

where $K=A[\xi] / L$ is a commutative $A$-algebra and thus from $i_{p}$ we obtain a monomorphism $A[X] / P \rightarrow M_{n}(K)$ which is an $A$-algebra monomorphism. This completes the proof of our lemma.

REMARK. (1) The method of proof is due to Procesi [2, Theorem 2.5] who has shown that $(*)$ holds for semiprime ideal $P$ and field $F=A$, since then $F[X] / P$ can be embedded in a matrix ring over a commutative ring.

(2) Note also that the order $n$ of the matrix ring $M_{n}(K)$ is the same as that of the generic matrices.

(3) If $A_{0}[X]$ is the subring of $A[X]$ generated by all polynomials in the generic matrices with free coefficient zero, then the preceding lemma and its proof remain valid also for ideals $P_{0}$ in $A_{0}[X]$, as long as embeddings of $A_{0}[X] / P_{0}$ as $A$-algebras are considered.

(4) If $A=Z$ the ring of integers, then every embedding of $Z_{0}[X] / P_{0}$ as well as $Z[X] / P$ is a $Z$-homomorphism.

The preceding lemma enables us to prove the following generalization of the Hilbert (basis) ascending chain condition theorem.

THEOREM 1. Let $A$ be a noetherian commutative domain, and $A[u]=A\left[u_{1}, u_{2}, \ldots, u_{r}\right]$ a finitely generated extension of $A$ (i.e. ap $[u]=p[u]$ a for every $a \in A, p[u] \in A[u]$ ), then $A[u]$ satisfies the following chain condition:

(**) Any nondecreasing sequence of ideals $P_{0} \subseteq P_{1} \subseteq \cdots \subseteq P_{k} \subseteq \cdots$ such that the rings $A[u] / P_{i}$ can be embedded as $A$-algebras in a matrix ring $M_{n_{i}}\left(K_{i}\right)$ with $K_{i}$ commutative and $\left\{n_{i}\right\}$ bounded-contains only a finite set of different ideals $P_{j}$.

Proof. Let $n$ be the greatest common multiple of all $n_{i}$. Consider the ring $A\left[X_{1}, \ldots, X_{r}\right]$ of the generic $n \times n$ matrices over $A$, and the homomorphism $\eta: A[X] \rightarrow A[u] / P_{0}$ given by $\eta\left(X_{i}\right)=u_{i}$. This is a well-defined homomorphism, since $A[u] / P_{0} \subseteq M_{n_{0}}\left(K_{0}\right)$ and $n_{0} \leqq n$-hence every relation $p[X]=0$ in $A[X]$ will yield $p[u] \equiv O\left(P_{0}\right)$. Let $\bar{P}_{i}=\eta^{-1}\left(P_{i}\right)$, then $\bar{P}_{i}$ are ideals in $A[X]$ and $\eta$ induces isomorphisms $A[X] / \bar{P}_{i} \cong\left(A[u] / P_{0}\right) /\left(P_{i} / P_{0}\right) \cong A[u] / P_{i}$. Now $A[u] / P_{i}$ can be embedded, by assumption, as an $A$-algebra in $M_{n_{i}}\left(K_{i}\right)$ and since $n$ is a multiple of the $n_{i}$ we clearly may assume $n_{i}=n$. Thus $A[X] / P_{i}$ has the same property and, therefore, it follows by the preceding lemma that $(*)$ holds, namely $\left\{\bar{P}_{i}\right\} \cap A[X]=\bar{P}_{i}$.

From this point we follow Procesi's proof of [2, Theorem 2.5].

Each of the ideals $\left\{\bar{P}_{i}\right\}$ generated by $\bar{P}_{i}$ in $M_{n}(A[\xi])$ is of the form $M_{n}\left(L_{i}\right)$ where 
$L_{i}$ is an ideal in $A[\xi]$. The sequence (**) implies that $\bar{P}_{i} \subseteq \bar{P}_{i+1}$ and therefore, we also have $\left\{\bar{P}_{i}\right\} \subseteq\left\{\bar{P}_{i+1}\right\}$ which yield a nondecreasing sequence $\left\{L_{i}\right\}$ of ideals in $A[\xi]$. The latter is a finitely generated polynomial ring over a noetherian domain and hence satisfies the ascending chain condition, which in our case implies that for some $m$ and all $j \geqq 0, L_{m}=L_{m+j}$. Hence $\left\{\bar{P}_{m}\right\}=\left\{\bar{P}_{m+j}\right\}$, which yield by $(*)$ that:

$$
P_{m}=A[X] \cap\left\{\bar{P}_{m}\right\}=A[X] \cap\left\{\bar{P}_{m+j}\right\}=P_{m+j} .
$$

The preceding proof requires that $A\left[u_{1}, \ldots, u_{n}\right]$ contain a unit element and so does $A$-this was assumed when $\eta$ was defined, but actually this is not necessary. One overcomes this difficulty by either considering the ring $A_{0}[X]$ of all polynomials in the generic matrices with zero free coefficient, or by adding a unit to $A\left[u_{1}, \ldots, u_{n}\right]$ and noting that this will not essentially change the assumption on the sequence $\left\{P_{i}\right\}$ of $(* *)$.

REMARK. The preceding theorem is an extension of one form of the Hilbert basis theorem; namely, the fact that the ascending chain condition hold for certain two-sided ideals, which for the commutative case are all ideals (though our method does not yield a new proof for this case). The last theorem raises some interesting questions:

It is well known that without the assumption that each $A[u] / P_{i}$ can be embedded in $M_{n_{i}}(K)$-the theorem is false. A simple counterexample is the free ring. Nevertheless one would expect that this would be true for the ring $A[X]$ of finite generic matrices. We shall prove that even this is false for matrices of order $n \geqq 2$. Thus, the Hilbert basis theorem for rings with polynomial identity is false even for rings which satisfy all identities of matrix rings. This already implies that it is even not sufficient to require that in (**) only $A[X] / P_{0}$ can be embedded in $M_{n}(K)$. We shall give an example to this effect.

Another question to which we have no answer is the validity of the other form of the Hilbert basis theorem. Namely, does each of ideals $P_{i}$ of $(* *)$ have a finite basis? This is true for maximal ideals as shown by Procesi [2].

3. Subrings of $M_{n}(K)$. In the following we shall use in $A[X]$ only two generic matrices and we prefer to denote them by $X=\left(\xi_{i k}\right)$ and $Y=\left(\eta_{i k}\right) i, k=1,2, \ldots, n$ and $A[\xi, \eta]$ will denote the ring of all commutative polynomials in the $2 n$ indeterminates $\xi_{i k}, \eta_{i k}$. Let $S_{k}\left[x_{1}, \ldots, x_{k}\right]=\sum \pm x_{i_{1}} x_{i_{2}} \cdots x_{i_{k}}$ denote the standard polynomial in $k$ noncommutative indeterminates $\left\{x_{i}\right\}$. Recall that the sum ranges over all permutation of $k$ letters with sign plus for even permutations and minus for odd permutations.

Consider the polynomials $p_{j}[X, Y]=S_{n}\left[Y^{n-1+j} X, Y^{n-2} X, Y^{n-3} X, \ldots, Y X, X\right]$ for $j \geqq 0$. Let $P_{0}$ be the ideal in $A[X, Y]$ generated by $p_{0}[X, Y]$ and similarly $P_{k}$ the ideal generated in $A[X, Y]$ by $p_{0}[X, Y], p_{1}[X Y], \ldots, p_{k}[X Y]$.

Our main result is the following

TheOREM 2. (a) The quotient ring $A[X Y] / P_{0}$ cannot be embedded (as an A-algebra) in any $M_{n}(K), K$ a commutative A-algebra. 
(b) Similarly the ideals $A[X, Y] / P_{k}$ cannot be embedded in any $M_{n+k}(K)$ with the same restrictions as in (a).

(c) The sequence $P_{0} \subset P_{1} \subset P_{2} \subset \ldots \subset P_{k} \subset \ldots$ is a strictly increasing sequence of ideals in $A[X, Y]$.

Before proceeding with the proof of the theorem we compute the polynomials $p_{k}[Y, X]$ for diagonal matrices:

Proposition. If $Y_{0}$ is the diagonal matrix with $y_{1}, y_{2}, \ldots, y_{n}$ in the diagonal then: $p_{k}\left[Y_{0}, X\right]=P[X] \cdot V_{k}\left(y_{1}, \ldots, y_{n}\right)$ where $P[X] \neq 0$ is a matrix depending on $X$ only, and

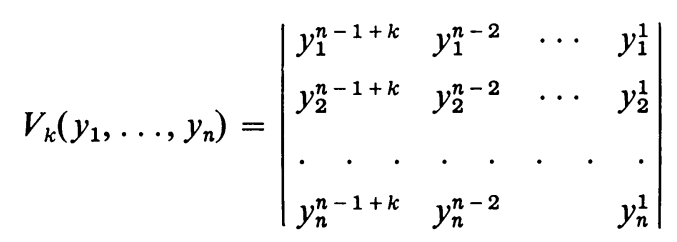

is a scalar depending on $Y_{0}$. In particular, $V_{0}\left(y_{1} \cdots y_{n}\right)=\prod_{i<j}\left(y_{i}-y_{i}\right)$ and $V_{k}\left(y_{1}, \ldots, y_{n}\right)=V_{0}\left(y_{1}, \ldots, y_{n}\right) \cdot s_{k}\left(y_{1}, \ldots, y_{n}\right)$ where $s_{k}$ is a symmetric polynomial in the $y_{i}$ 's.

Proof. The entry in the $(\lambda, \mu)$ place of the matrix

$$
p_{k}\left[X, Y_{0}\right]=S_{n}\left[Y_{0}^{n-1+k} X, Y_{0}^{n-2} X, \ldots, Y_{0}\right]
$$

is the element:

$$
\pi_{\lambda \mu}^{k}=\sum_{(i)} \sum_{(\lambda)} \sigma_{(i)} y_{\lambda}^{i_{1}} \chi_{\lambda \lambda_{1}} y_{\lambda_{1}}^{i_{2}} \chi_{\lambda_{1} \lambda_{2}} \cdots y_{\lambda_{n-2}}^{i_{n-1}} \chi_{\lambda_{n-2} \lambda_{n-1}} y_{\lambda_{n-1}}^{i_{n}} \chi_{\lambda_{n-1} \mu},
$$

where the first sum ranges over all permutations $\left(i_{1}, \ldots, i_{n}\right)$ of $(n-1+k, n-2, \ldots$, $1,0)$ with the appropriate sign $\sigma_{(i)}= \pm 1$ and the sum ranges over all $\lambda_{j}=1,2, \ldots, n$ for all $j=1,2, \ldots, n-1$. Reversing the order of summation we have

$$
\pi_{\lambda \mu}=\sum_{(\lambda)} \bar{P}\left(\lambda, \lambda_{1}, \ldots, \lambda_{n-1}, \mu\right) \sum_{(i)} \sigma_{(i)} y_{\lambda}^{i_{1}} y_{\lambda_{1}}^{i_{2}} \cdots y_{\lambda_{n-1}}^{i_{n}} .
$$

The term with the $y$ 's is by definition of determinants $V_{k}\left(y_{\lambda}, y_{\lambda_{1}}, \ldots, y_{\lambda_{n-1}}\right)$ where $V_{k}$ was defined in our proposition for $y_{1}, \ldots, y_{n}$. Clearly, $V_{k}\left(y_{\lambda}, y_{\lambda_{1}}, \ldots, y_{\lambda_{n-1}}\right)=0$ if any two of the $y$ 's are equal, and otherwise

$$
V_{k}\left(y_{\lambda}, y_{\lambda_{1}}, \ldots, y_{\lambda_{n-1}}\right)=\sigma_{(\lambda)} \cdot V_{k}\left(y_{1}, y_{2}, \ldots, y_{n}\right)
$$

where $\sigma_{(\lambda)}= \pm 1$ is the sign of the permutation

$$
\left(\begin{array}{llll}
1 & 2 & \cdots & n \\
\lambda & \lambda_{1} & \ldots & \lambda_{n-1}
\end{array}\right) .
$$

Thus we have

$$
\pi_{\lambda \mu}^{k}=V_{k}\left(y_{1}, \ldots, y_{n}\right) \cdot \sum_{\left(\lambda_{1}\right)}^{*} \sigma_{\left(\lambda_{t}\right)} \chi_{\lambda \lambda_{1}} \chi_{\lambda_{1} \lambda_{2}} \cdots \chi_{\lambda_{n-1} \mu}=V_{k} P_{\lambda \mu}(x)
$$


where $\Sigma^{*}$ ranges over all permutation $\left(\lambda \lambda_{1} \cdots \lambda_{n-1}\right)$ of $(12 \cdots n)$ with $\lambda$ fixed and $\lambda_{i}=1,2, \ldots, n$, and $\sigma_{\left(\lambda_{i}\right)}= \pm 1$ the sign of the permutation. This proves the first part of our proposition with $P=\left(P_{\lambda \mu}\right)$.

To prove that $P[X] \neq 0$, we substitute for $X$ the matrix

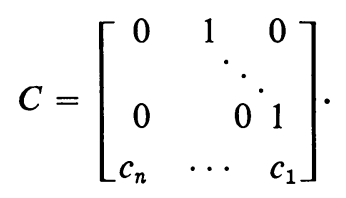

One readily observes that in a monomial of the form $Z_{1} C Z_{2} C \cdots Z_{j} C$ where the $Z_{i}$ are diagonal matrices, the first $n-j+1$ rows are the same as that of $C^{j}$ but each entry multiplies by a monomial in the coefficient of the $Z_{i}$. In particular, since we know that $p_{k}\left[Y_{0}, C\right]=V_{k}(y) P[C]$ it follows that the first row of $P[C]$ is the same as that of $C^{n}$, namely $\left(c_{n} c_{n-1} \cdots c_{1}\right)$ which is not zero. The second part is well known, but for the sake of completeness we indicate a proof that: $V_{k}=V_{0} s_{k}$ with $s_{k}$ symmetric. Indeed, the $y_{i}$ satisfy the equation $y^{n}-c_{1} y^{n-1}+\cdots+(-1)^{n} c_{n}$ $=\prod\left(y-y_{i}\right)$ with the $c_{i}$ the elementary symmetric polynomials. This readily implies that each $y=y_{i}$ satisfy a relation $y^{n-1+k}=s_{k} y^{n-2}+\cdots$ where the other terms are of the form $a_{i} y^{i}, 0 \leqq i<n-2$ and $a_{i}$ symmetric. Substituting this presentation of each $y_{i}^{n-1+k}$ in $V_{k}$ yields our result.

A more general computation of this type will be used elsewhere to obtain a relation between symmetric functions and identities of matrices.

REMARK. The computations involved in our proof can be readily used to prove the following:

Let $Y_{i}=\sum_{k} y_{i k} c_{k k}$ be diagonal matrices then

$$
S_{n}\left[Y_{1} X, Y_{2} X, \ldots, Y_{n} X\right]=P[X] \operatorname{det}\left|y_{i k}\right| \text {. }
$$

We are now in positition to give the:

Proof of Theorem 2. In view of Lemma 1 it suffices to show that $\left\{P_{0}\right\} \cap A[X, Y]$ $\neq P_{0}$ in the ring of $n \times n$ generic matrices. Indeed, we shall show that $p_{1}[Y, X] \in\left\{P_{0}\right\}$ $\cap A[X, Y]$ but $\notin P_{0}$. To prove the first assertion, we note that $Y$ satisfies its characteristic polynomial $Y^{n}-t_{1} Y^{n-1}+\cdots=0$. Hence

$$
\begin{aligned}
p_{1}[X, Y] & =S_{n}\left[Y^{n} X, Y^{n-2} X, \ldots\right]=\sum_{i=1}^{n}(-1)^{i-1} t_{i} S_{n}\left[Y^{n-i} X, Y^{n-2} X, \ldots, X\right] \\
& =t_{1} S_{n}\left[Y^{n-1} X, \ldots\right]=t_{1} p_{0}[X, Y]
\end{aligned}
$$

since the other terms have two equal entries in $S_{n}[\cdot]$ and hence they are zero. Now, $t_{1}=$ trace $Y=\sum y_{i i}$, so that $p_{1}[Y, X] \in\left\{P_{0}\right\} \cap A[X, Y]$.

Next $p_{1} \notin P_{0}$. If this were not the case then $p_{1}=\sum a_{\lambda} p_{0} b_{\lambda}$ where $a_{\lambda}, b_{\lambda} \in A[X, Y]$. This will yield a relation

$$
q[X, Y]=p_{1}[X, Y]-\sum a_{\lambda}[X, Y] p_{0}[X Y] b_{\lambda}[X, Y]=0
$$


which vanish for the generic matrices and hence vanish in all $M_{n}(K)$ for commutative $K \supseteq A$. Hence, each homogeneous part of $q[X, Y]$ (in $Y$ or $X$, or both) vanishes identically. $p_{1}, p_{0}$ are of degree $n$ in $X$, and their degree in $Y$ differ in 1 so by taking the homogeneous part of the polynomial $q$ we get a relation

$$
p_{1}[X, Y]-a Y p_{0}[X, Y]-b p_{1}[X, Y] Y=0 \text { for some } a, b \in A \text {. }
$$

But no identity (R) can hold in $M_{n}(K)$ for any $K \supseteq A$. Indeed, substitute for $Y$ the diagonal matrix $Y_{0}$ then we obtain from (R) by our proposition that $P[X] s_{1} V_{0}(y)-a Y P[X] V_{0}(y)-b P[X] Y V_{0}(y)=0$. The first row of $P[X]$ contains nonzero elements, hence by computing the element in the $(1 j)$ place of our last relation, we readily obtain that $s_{1}-a y_{1}-b y_{j}=0$, where $s_{1}$ is symmetric $y_{1}, \ldots, y_{n}$ and the relation holds for all $j$ which is clearly impossible (since $s_{1} \not \equiv 0$ ). This completes the proof of (a).

The proof of (b) follows similar lines but needs some important modifications:

Let $A[\bar{X}, \bar{Y}]$ be the ring of generic matrices of order $n+k$ over $A$, and $A[x, y]$ the free polynomial ring in noncommutative indeterminate $x, y$ commuting with the element of $A$. Let $\rho: A[x, y] \rightarrow A[X, Y]$ and $\bar{\rho}: A[x, y] \rightarrow A[\bar{X}, \bar{Y}]$ given by $\rho(x)=X, \rho(y)=Y$ and similarly $\bar{\rho}(x)=\bar{X}, \bar{\rho}(y)=\bar{Y}$. Since every relation of $f(x, y)=0$ which holds for generic matrices of order $n+k$ will hold also for generic matrices of order $n$, it follows that $\operatorname{Ker} \bar{\rho} \subseteq \operatorname{Ker} \rho$. Hence there exist a homomorphism over $A, \sigma: A[\bar{X}, \bar{Y}] \rightarrow A[X, Y]$ such that $\sigma(\bar{X})=X$ and $\sigma(\bar{Y})=Y$. If $P_{k}$ is the ideal generated by $p_{0}(X, Y), \ldots, p_{k}(X, Y)$ in $A[X, Y]$ then we denote by $\bar{P}_{k}=\sigma^{-1}\left(P_{k}\right)$ and $\sigma$ induces an isomorphism $A[\bar{X}, \bar{Y}] / \bar{P}_{k} \cong A[X, Y] / P_{k}$. Note that $\bar{P}_{k}$ is generated by $p_{0}(\bar{X}, \bar{Y}), \ldots, p_{k}(\bar{X}, \bar{Y})$ and $\operatorname{Ker} \sigma$ which is the set of all $f(\bar{X}, \bar{Y})$ which vanish for $n \times n$ matrices. Finally, we prove that $A[\bar{X}, \bar{Y}] / \bar{P}_{k}$ cannot be embedded in any $M_{n+k}(K)$ by utilizing Lemma 1 ; namely, it will be shown that $\left\{\bar{P}_{k}\right\} \cap A[\bar{X}, \bar{Y}] \neq \bar{P}_{k}$. This is achieved by proving that $p_{k+1}(\bar{X}, \bar{Y}) \in\left\{\bar{P}_{k}\right\} \cap A[\bar{X}, \bar{Y}]$ and $\notin \bar{P}_{k}$.

Indeed, $\bar{Y} \in M_{n+k}(A[\xi, \eta])$ hence $\bar{Y}$ satisfy a polynomial relation $\bar{Y}^{n+k}=$ $\sum_{i=1}^{n+k} t_{i} Y^{n+k-i}$, furthermore the coefficients $t_{i}$ are polynomials in the entries of $\bar{Y}$, i.e. $t_{i} \in A[\xi, \eta]$. Hence

$$
\begin{aligned}
p_{k+1}(\bar{X}, \bar{Y}) & =S_{n}\left[\bar{Y}^{n+k} \bar{X}, \bar{Y}^{n-2} \bar{X}, \ldots, \bar{X}\right]=\sum_{i=1}^{n+k} t_{i} S_{n}\left[\bar{Y}^{n+k-i} \bar{X}, \bar{Y}^{n-2} \bar{X}, \ldots, \bar{X}\right] \\
& =\sum_{i=1}^{k-1} t_{i} S_{n}[\cdot]=\sum_{i=1}^{k-1} t_{i} P_{k-i+1}(\bar{X}, \bar{Y}) \in\left\{\bar{P}_{k}\right\} \cap A[\bar{X}, \bar{Y}] .
\end{aligned}
$$

Next if $p_{k+1}(\bar{X}, \bar{Y}) \in \bar{P}_{k}$, then we have a relation

$$
p_{k+1}(\bar{X}, \bar{Y})+\sum a_{\lambda i}(\bar{X}, \bar{Y}) p_{i}(\bar{X}, \bar{Y}) b_{\lambda i}(\bar{X}, \bar{Y})+g(\bar{X}, \bar{Y})=0
$$

where $g(\bar{X}, \bar{Y}) \in \operatorname{Ker} \sigma$ and $i=0,1, \ldots, k$. Hence if we apply $\sigma$ on $\left(\mathrm{R}_{k}\right)$ we get

$$
q(X, Y)=p_{k+1}(X, Y)+\sum_{\lambda, i} a_{\lambda i}(X, Y) p_{i}(X, Y) b_{\lambda i}(X, Y)=0 .
$$

Again by the argument on the homogeneous part of $q(X, Y)$, as in the proof of 
part (a), it follows that $a_{\lambda i}=\varphi_{\lambda i}(Y), b_{\lambda i}=\psi_{\lambda i}(Y)$ are polynomials in $Y$ only and $\operatorname{deg}_{Y} a_{\lambda i}+\operatorname{deg}_{Y} b_{\lambda i}=k-i$ since $\operatorname{deg}_{Y} p_{k+1}-\operatorname{deg}_{Y} p_{i}=k-i$.

Substituting the diagonal matrix $Y_{0}$ for $Y$, and applying Lemma 1 we have

$$
P[X] \cdot V_{k+1}(y)+\sum \varphi_{\lambda i}(Y) P[X] \psi_{\lambda i}(Y) V_{i}(y)=0 .
$$

Again computing the elements in the $(1 j)$ place of this matrix and noting that the entries of $P[X]$ are nonzero we obtain a relation:

$$
V_{k+1}(y)+\sum \varphi_{\lambda i}\left(y_{1}\right) \psi_{\lambda i}\left(y_{j}\right) V_{i}(y)=0
$$

for $j=1,2, \ldots, n$ and the sum ranges for some $\lambda$, but $i$ ranges over $0,1, \ldots, k$ and also deg $\varphi_{\lambda i}+\operatorname{deg} \psi_{\lambda i}=k-i$ for all possible $\lambda$. This leads in view of our proposition that:

$$
s_{k+1}\left(y_{1}, \ldots, y_{n}\right)+\sum_{\lambda} \sum_{i=0}^{k} \varphi_{\lambda i}\left(y_{1}\right) \psi_{\lambda i}\left(y_{i}\right) s_{i}\left(y_{1} \cdots y_{n}\right)=0
$$

and our contradiction will be obtained by showing that no relation of the type $\left(\mathrm{R}_{k}\right)$ can exist between the $s_{i}$. To this end we shall show first that for each $i$, $s_{i}\left(y_{1}, \ldots, y_{n}\right)$ is homogeneous of degree $n+i$ and has the term $y_{1}^{n+i}$ as well as $y_{2}^{n+i}$ among its monomials: It follows readily from the determinant form of $V_{i}\left(y_{1} \cdots y_{n}\right)$ of our proposition that it is homogeneous, hence, since $V_{i}=V_{0} s_{i}$ it follows that $s_{i}(y)$ is homogeneous. The polynomial $V_{i}(y)$ has a nonvanishing term $y_{1}^{n+i-1} y_{2}^{n-2} y_{3}^{n-1} \cdots y_{n-1}$ and $V_{0}(y)$ has the term $y_{1}^{n-1} y_{2}^{n-2} \cdots y_{n}^{0}$. From a lexicographically ordering argumentation it follows clearly since $s_{i}$ is of degree $i$ (and symmetric) that the relation $V_{i}=V_{0} s_{i}$ implies that $s_{i}(y)=y_{1}^{i}+y_{2}^{i}+\cdots$.

Next consider the polynomial in a new indeterminate $z$ given by $\left(\mathbf{R}_{k}\right)$ :

$$
u(z)=s_{k+1}\left(y_{1} \cdots y_{n}\right)+\sum_{\lambda} \sum_{i=0}^{k} \varphi_{\lambda i}\left(y_{1}\right) s_{i}\left(y_{1}, \ldots, y_{n}\right) \psi_{\lambda i}(z)
$$

then $u(z)$ vanishes for all $y_{i}$. By a straightforward division of $u(z)$ by $z-y_{1}, z-y_{2}$, $\ldots, z-y_{n}$ it follows readily that $u(z)$ is divisible in $A\left[y_{1}, \ldots, y_{n}\right]$ [Z] by the polynomial $\prod_{i=1}^{n}\left(z-y_{i}\right)=g(z)$. But no relation of the type $u(z)=g(z) h(z)$ where the coefficient of $h(z)$ are polynomials in $y$ exists. For, set $z=0$ and obtain a relation

$$
s_{k+1}\left(y_{1}, \ldots, y_{n}\right)+\sum \varphi_{\lambda i}\left(y_{1}\right) s_{i}\left(y_{1} \cdots y_{n}\right) \psi_{\lambda_{i}}(0)=m\left(y_{1} \cdots y_{n}\right) y_{1} y_{2} \cdots y_{n}
$$

where $m$ is a polynomial in $y_{i}$. From the fact that $\varphi_{\lambda i}$ and $\psi_{\lambda i}$ are homogeneous and $\operatorname{deg} \varphi_{\lambda i}+\operatorname{deg} \psi_{\lambda i}=k+1-i$ it follows that $\psi_{\lambda i}(0)=0$ unless $\operatorname{deg} \varphi_{\lambda i}=k+1-i$ so our last relation can be put in the form

$$
s_{k+1}\left(y_{1}, \ldots, y_{n}\right)+\sum_{i=1}^{k} a_{i} y_{i}^{k+1-i} s_{i}\left(y_{1}, \ldots, y_{n}\right)=m\left(y_{1} \cdots y_{n}\right) y_{1} \cdots y_{n} .
$$

The coefficient of $y_{2}^{k+1}$ on the right side is by our previous remark equal to 1 , coming only from $s_{k+1}\left(y_{1} \cdots y_{n}\right)$ since the other will have $y_{1}^{k+1-i}$ and $k+1-i>0$. 
But, this is impossible since the right side is a multiple of $y_{1} y_{2} \cdots y_{n}$. This completes our contradiction, so that $p_{k+1}(X, Y) \notin P_{k}$ and the validity of (b) is obtained.

In fact the preceding proof yields also (c). For, $p_{k+1} \notin P_{k}$ but $p_{k+1}$ is one of the generators of $P_{k+1}$-thus $P_{k} \neq P_{k+1}$ and clearly $P_{k} \subset P_{k+1}$.

4. Remarks and examples. The quotient rings $A[X, Y] / P_{k}$ yields also interesting examples of rings.

THEOREM 3. For $n \geqq 3$, there exists rings which are homomorphic images of subrings of $n \times n$ matrices over commutative rings, and satisfies exactly the identities of $M_{n}(Z)$ ( $Z$-the rings of integers) but nevertheless cannot be embedded in any $M_{r}(K)$ for any finite $r$ and any commutative ring $K$.

Proof. Consider the ring $Z[X, Y] / P_{k}=\mathscr{R}_{k}$ the $\mathscr{R}_{k}$ is a homomorphic image of $Z[X, Y]$ which is a subring of $M_{n}\left(Z\left[\xi_{i k}, \eta_{i k}\right]\right)$. Thus $\mathscr{R}_{k}$ satisfies all identities of $M_{n}(Z)$, and to show that these are exactly the identities of $\mathscr{R}_{k}$ we prove for the case $n \geqq 3$ the existence of a homomorphic image which is almost the complete $M_{n}(Z)$, and in any case satisfies only the identities of $M_{n}(Z)$.

Consider the homomorphism of $\tau: Z[X, Y] \rightarrow M_{n}(Z)$ given by

$$
X \rightarrow\left(\begin{array}{ccc}
v_{1} & & 0 \\
& \ddots & \\
0 & & v_{n}
\end{array}\right)=V
$$

where $v_{i}$ are different elements of $\boldsymbol{Z}$ and

$$
Y \rightarrow\left(\begin{array}{llll}
1 & 1 & \cdots & 1 \\
1 & 1 & \cdots & 1 \\
\hdashline 1 & 1 & \cdots & 1
\end{array}\right)=U
$$

Then $U^{2}-n U=0$, hence $p_{t}(U, V)=S_{n}\left[U^{n+t-1} V, U^{n-2} V, \ldots, U V, V\right]=0$. Since $U^{i}=a_{i} U$ for $i \geqq 2$ and so if $n-2 \geqq 1$ (i.e. $n \geqq 3$ ), two of the entries in $S_{n}[\cdots]$ are linearly dependent and so $S_{n}[\cdots]=0$. The image of $\tau$ which is the $\operatorname{ring} Z[U, V]$ will contain all elements of the form $m_{\alpha \beta} C_{\alpha \beta}$ for some $0 \neq m_{\alpha \beta} \in Z$ and where $C_{\alpha \beta}$ is the matrix with 1 in $\alpha$ th row and $\beta$-column. Indeed, consider the polynomial $f_{\alpha}=\sum a_{t \alpha} \lambda^{t}=\prod_{i \neq \alpha}\left(\lambda-v_{i}\right)$ then $f_{\alpha}(V)=m_{\alpha} C_{\alpha \alpha}, m_{\alpha} \neq 0$ and hence $f_{\alpha}(V) U f_{\beta}(V)=$ $m_{\alpha} m_{\beta} C_{\alpha \beta}$ as required.

Thus $\tau(Z[U, V]) \otimes_{R} Q=M_{n}(Q)$ where $Q$ is the field of all rationals, and thus $\tau(Z[U, V])$ satisfies exactly the same identities as that of $M_{n}(Q)$ which are the same as those of $M_{n}(Z)$. Q.E.D.

It follows by Theorem 2 that $Z[X, Y] / P_{k}$ cannot be embedded in any $M_{n+k}(K)$ since for $A=Z$ any embedding is a $Z$-algebra embedding.

Finally, the direct sum $\sum\left(Z[X, Y] / P_{k}\right)=\mathscr{R}$ is clearly a ring satisfying Theorem 3 .

The preceding results lead to the interesting question of finding necessary and 
sufficient conditions for a ring $R$ to be embeddable in a matrix ring $M_{n}(K)$ over a commutative ring $K$. It follows from our result that the validity of the identities of a matrix ring by $R$ is only a necessary condition but not sufficient. The following theorem gives another necessary condition for finitely generated rings, and the problem we propose: are those two conditions together necessary and sufficient.

THEOREM 4. Let $R=F\left[w_{1}, \ldots, w_{r}\right]$ a finitely generated $F$-algebra where $F$ is a field, which can be embedded in some $M_{n}(K)$ as an F-algebra, then the Jacobson radical $J(R)$ of $R$ is nilpotent.

Proof. As in the proof of Lemma 1, we have an isomorphism $F\left[X_{1}, \ldots, X_{2}\right] / P$ $\cong F\left[u_{1}, \ldots, u_{r}\right]$ for some ideal $P$ is the ring of $n \times n$ generic matrices $F[X]$, and $P$ satisfies condition $(*)$ of Lemma 1 . As in the proof of that lemma we have an embedding of $F[X] / P$ into $M_{n}(F[\xi] / L)$ where $L$ is the ideal in $F[\xi]$ generated by all entries of the matrices of $\{P\}$. Let $J / L$ be the Jacobson radical of $F[\xi] / L$.

If $U$ is a nil subring of $M_{n}(F[\xi] / L)$ then $U$ is nil $\bmod M_{n}(F[\xi] / J)$. Now $F[\xi] / J$ is a subdirect sum of field and the nil subrings of matrices over fields are nilpotent of index $n$, hence $U^{n} \subseteq M_{n}(J / L)$. Now $J$ is nilpotent modulo $L$, as the Jacobson radical in a finitely generated commutative algebra. Thus $U^{n m}=0$ for some $m$.

The final step of the proof is the observation that the Jacobson radical $J(R)$ for finitely generated algebras which satisfy an identity is nil [1]. Hence $J(R)^{n m}=0$. Q.E.D.

\title{
BIBLIOGRAPHY
}

1. S. A. Amitsur and C. Procesi, Jacobson-rings and Hilbert algebras with polynomial identities, Ann. Mat. Pura Appl. (4) 71 (1966), 61-72. MR 34 \#5869.

2. C. Procesi, Non-commutative affine rings, Atti Accad. Naz. Lincei Mem. Cl. Sci. Fis. Mat. Natur. Sez. I (8) 8 (1967) 237-255. MR 37 \#256.

\author{
HeBREW UNIVERSITY, \\ JERUSALEM, ISRAEL \\ YALE UNIVERSITY, \\ New Haven, Connecticut 06520
}

